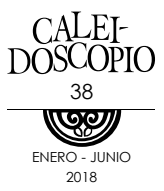

\title{
Trayectoria profesional femenina: contrastando modelos
}

Female career path: contrasting models

CLARA SELVA OLID'

FRANCISCO JAVIER RUVALCABA COYASO²

\section{RESUMEN}

La trayectoria profesional femenina sigue siendo una temática de interés recurrente. Esto se explica por la multiplicidad de ámbitos de investigación, aproximaciones metodológicas y ópticas que presenta su estudio. El objetivo de este artículo es revisar los modelos de carrera identificados en la literatura especializada respecto al desarrollo profesional de la mujer hacia cargos directivos, contrastarlos y abordar las limitaciones más esgrimidas en estos trayectos. Tras la revisión realizada, los resultados apuntan a cuatro grandes agrupaciones o modelos de carrera que explican las trayectorias de la mujer hacia los cargos de dirección y una clasificación de desarrollo aplicable a cada uno de ellos. Como principal aportación, este trabajo articula los modelos de carrera presentes en el corpus literario, acota y vincula las limitaciones más presentes en la realidad laboral y rompe con caducas creencias que operan en el mundo profesional respecto a las trayectorias de las mujeres.

Palabras clave: reinterpretación de datos, mujeres, trayectoria profesional, modelos de carrera, dirección.

1 Universitat Autònoma de Barcelona

2 Universidad Autónoma de Aguascalientes

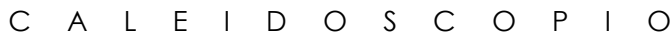


The professional career of women keeps being a thematic of greater and recurrent interest; this is explained by the multiplicity of researching areas, methodological approaches and perspectives that this topic presents. The goal of this article is to review the career models identified in specialized literature regarding professional development of women towards management positions, to approach and contrast the limitations provided in these paths. After the revision of the literature, the results show four big groups or career models that explain the female career path towards management positions and a growth classification applicable to each of them. As a main contribution, this paper articulates the models of career present in the literary corpus, bounds and links the most common limitations in the labor reality as well as breaks with out of date convictions that operate in the professional world about female career paths.

Keywords: data reinterpretation, women, professional career, models of career, management.

\section{TRAYECTORIA PROFESIONAL FEMENINA: AOUÍ Y HOY}

Inmersos en pleno siglo XXI y con los avances socioculturales acaecidos en las últimas décadas -como la irrupción de las nuevas tecnologías de la información y la comunicación o la constante innovación y transformación social y organizativa-, sorprende que la trayectoria profesional femenina siga siendo una temática de candente interés; abordada desde las experiencias de las propias protagonistas, dándoles voz, hasta las posiciones más de intervención en las que se sitúan profesionales, investigadores y organizaciones de distinta índole. La relevancia de la trayectoria profesional se explica por una conjunción de factores, entre ellos destaca el abordaje multidisciplinar que presenta (estudiada desde la sociología, la educación, la historia o la psicología), su interconexión, metodologías y complejidad; lo que plantea todo un reto a las personas interesadas en su estudio: dar respuestas a un fenómeno de múltiples ópticas, ámbitos y realidades profesionales.

Diversas razones explican la lejanía o limitaciones que caracterizan los caminos de la mujer, en relación a su homónimo masculino, respecto a los cargos más altos de la organización (Freixas, 2004). Las 
teorías más citadas sobre limitaciones femeninas en el mundo laboral versan sobre: el capital cultural de las familias, los estereotipos sociales o la masculinización de ciertas profesiones (Heilman, 2001). Estas limitaciones, como apuntan otros autores (Wajcman, 2004; Goyette y Mullen, 2006), nos hacen pensar en que las mujeres se sienten menos atraídas por profesiones donde se visualizan mayores dificultades o un clima poco favorable para su desarrollo profesional y personal.

Otras teorías (Baltres-Löhr, 2006) señalan como principales limitaciones las estructuras y culturas organizativas predominantemente jerárquicas, así como los primeros momentos de socialización organizacional que exigirían ritmos de trabajo y exigencias profesionales incompatibles con la conciliación de los otros espacios -personal y familiar. Mientras que, otras tantas teorías (Fernandez y Mors, 2008; Molero, Cuadrado, García-Ael, Recio y Rueda, 2009) señalan la escasa presencia de las mujeres en los órganos de decisión y su menor presencia en las redes sociales. Éstas, muy masculinizadas, harían de órgano estructurador, gestionando y perpetuando la reproducción homosocial en las organizaciones (Grimmer y Röhl, 2005).

Entre las limitaciones de nuestra era sobresalen la cultura empresarial y la conciliación de espacios, sin obviar aquellas que persisten de era en era, como los estereotipos (Selva, 2012). Por tanto, a pesar de los múltiples cambios y transformaciones sociales, las mayores desigualdades siguen acusándose en el momento de incorporación al trabajo y en la promoción profesional, donde la cultura organizativa juega un papel primario. Estando la cultura anclada en los clásicos modelos y roles patriarcales eminentemente masculinos, parece obvio que sólo perpetúe la reproducción homosocial en las estructuras organizativas (Wajcman, 1998; Wajcman y Martin, 2002), relegando a las mujeres a las posiciones de cargo medio como su techo de cristal (Cuadrado y Morales, 2007; Linehan, 2000). Así, como apuntan Bozeman, Dietz y Gaughan (2001), lograr una carrera profesional de éxito no depende sólo de ser brillante, sino también de las condiciones sociales o políticas imperantes en la organización, es decir, de su cemento cultural (González, 2009).

A pesar de las medidas y la legislación propuesta contra la discriminación -que incluyen la acción positiva o las iniciativas de diversidad-, así como el crecimiento significativo del número de mujeres en los puestos de gestión, la realidad sigue mostrando que la mayoría de los puestos de alta dirección siguen dominados por hombres

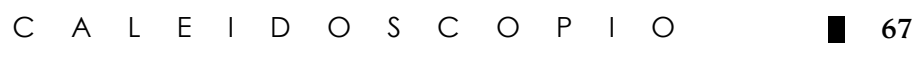


(López y García-Retamero, 2009), y que las culturas organizativas son predominantemente masculinas (Simpson, 2000; Wajcman, 1998; Wajcman y Martin, 2002). Por todo ello, es posible afirmar que la igualdad aún está lejos de los puestos de alta dirección. A esto debe sumarse la tríada "maternidad, familia y trabajo" que, lejos de resolverse, continúa llevando nombre de mujer (Carosio, 2010).

Todas estas teorías y limitaciones son recogidas por diferentes categorizaciones, desde aquellas que tienen en cuenta el locus de control: sea este interno -como la baja autoeficacia respecto a su homónimo masculino o la formación en áreas tradicionalmente femeninas (Agut y Hernández, 2007; Barberá, Ramos y Sarrió, 2000)-; o externo -menores oportunidades en relación al desarrollo de su trayectoria profesional o la ausencia de políticas reales que permitan la conciliación de espacios (Cuadrado y Morales, 2007; Sarrió, Barberá, Ramos y Candela, 2002)-; hasta las que ponen el acento en el origen de estos factores: entorno, organización o individuo. Identificando así tres grupos: contextuales, como la masculinización de la cultura de la organización o el sector de actividad que desarrolla; empresariales, como el tamaño de la empresa, la edad o el acceso a la financiación; y los intrínsecos a la mujer, como los aspectos de personalidad o los vínculos familiares (Junquera, 2004). Sin olvidar aquellas que presentan las barreras en relación al ciclo profesional: antes, después o más allá del después. Entendiendo las barreras antes como las anteriores a la entrada al mundo laboral; las barreras después, como las relacionadas con el acceso al trabajo y su desempeño; y las más allá del después, como las que abordan las limitaciones de las mujeres que se reincorporan al trabajo después de un lapso de tiempo -e.g. la maternidad- (Padilla, 2001).

Sea cual fuere la clasificación, lo que queda claro es que siguen existiendo frenos que entorpecen la llegada de la mujer a los cargos de dirección a pesar de: a) la masiva incorporación de la fuerza laboral femenina al ámbito profesional (López, 2007); 6) haber superado las barreras asociadas a la menor formación (Bonilla y Martínez, 1992) -ellas se licencian más y con mejores notas que sus colegas masculinos (Instituto Nacional de Estadística, 2016)-; y c) de encontrarnos en plena vorágine de transformación social, por la que deberían haber quedado obsoletos muchos de los estereotipos sociales más enraizados en nuestra cultura (Baron y Byrne, 2005; Dickman e Eagly, 2000; Ridgeway, 2001). Estos frenos no solamente suponen un perjuicio para las mujeres que desean desarrollar su carrera profesional, sino 
también para el propio sistema, ya que no se trata sólo de discriminación, sino también de un flagrante derroche del talento (González, 2009; Moreno, 2016).

Los datos presentados en torno a la situación de la mujer en el mundo laboral y los referentes a su posición en cargos directivos suponen un cuestionamiento a la retórica de la igualdad. Como señalan Selva, Tresserra, Pallarès y Sahagún (2012), a pesar de las medidas propuestas -la legislación existente contra la discriminación, la acción positiva, las iniciativas de diversidad y el crecimiento significativo en número de mujeres en lugares de gestión-, los puestos de dirección siguen, mayoritariamente, dominados por hombres (López y García-Retamero, 2009) en culturas e identidades organizativas predominantemente masculinas (Simpson, 2000; Wajcman, 1998; Wajcman y Martin, 2002). Por ello, es posible afirmar que la realidad de la igualdad está todavía lejos de los puestos de alta dirección.

Ante un contexto laboral no análogo para hombres y mujeres, este estudio pretende, desde un plano teórico, conocer qué trayectorias o mapas de ruta son aquellos que pueden ayudar a la mujer a llegar a los cargos de dirección -si es ese su anhelo. Específicamente el objetivo es revisar las tipologías de trayectoria o modelos de carrera identificados en la literatura especializada respecto al desarrollo profesional de la mujer, contrastarlos y atender a las limitaciones más esgrimidas en estos trayectos.

MAPAS DE RUTA: MÚLTIPLES CAMINOS PARA UNA REALIDAD LLENA DE POSIBILIDADES

La revisión de literatura realizada en las bases de datos: EBSCO, Dialnet, Academia.edu, Research Gate y Scholar Google, con combinatorias de palabras clave de primer nivel (modelos, clasificaciones, agrupaciones y arquetipos) y de segundo nivel (carrera profesional, trayectoria profesional y desarrollo profesional), arrojó 15 textos en los que, de una forma u otra, se remite a modelos, prototipos $u$ arquetipos de carrera, trayectoria o desarrollo profesional.

Los artículos revisados muestran que las mujeres siguen diferentes recorridos y trayectorias para llegar a ocupar cargos de dirección, desbancando así una vieja creencia de que sólo había un camino para llegar a la dirección, metaforizado por una escalera vertical o de ca- 
racol con una infinidad de peldaños (Cemborain, 2007). Una de las secuencias clásicas de avance profesional es la propuesta por Super (1962; 1963; 1976), quien sostuvo la existencia de diferentes tipologías de desarrollo laboral: a) el seguido por personas que se estabilizan pronto; b) el seguido por las que se estabilizan un poco más tarde; y c) el de otras tantas, que nunca llegan a hacerlo. En este sentido, Huberman, Thompson y Weiland (2000: 56) describen el desarrollo profesional como: "Un proceso en lugar de ser una serie sucesiva de hechos puntuales. Para algunas personas, este proceso puede parecer lineal, pero para la mayoría supone avances, regresiones, caminos sin salida y cambios de dirección imprescindibles desencadenados por nuevos acontecimientos: en resumen discontinuidades".

Antes de explorar las tipologías y/o agrupaciones de desarrollo de la mujer categorizadas en la literatura, cabría hacer referencia al concepto de carrera. Desde ámbitos científicos de estudio, la carrera profesional se entiende como un instrumento que permite reconocer las competencias profesionales de los trabajadores, así como el compromiso de las mismas respecto a la organización (Simó y Casado, 2006); mientras que desde ámbitos sociales, se entiende como el resultado de las transiciones que configuran la trayectoria profesional en relación al espacio histórico y social, en el que se transita por diferentes posiciones o roles profesionales (Selva, 2012). Por tanto, desde ámbitos científicos y sociales, se converge en entender la carrera como una secuencia de desarrollo profesional que permite conocer el espacio personal, social e histórico que rodea a la persona en cada una de las transiciones experimentadas.

Del total de textos seleccionados, la revisión en profundidad realizada atendiendo a tipologías y agrupaciones de trayectorias y carreras profesionales deja a la luz: a) cuatro grandes modelos, b) una clasificación de desarrollo aplicable a cada uno de ellos, y c) otros modelos de carrera situados en contextos específicos, como es el caso del deportivo.

De forma pormenorizada, los diferentes modelos, clasificaciones o agrupaciones halladas en la literatura remiten recurrentemente a cuatro esencias distintas en el desarrollo de la carrera profesional. Específicamente, nos referimos a cuatro grandes modelos que recogen una variada amalgama de autores, postulados y tipologías de desarrollo. A continuación, serán abordados atendiendo, en primera instancia, a sus secuencias características de desarrollo y, seguidamente, a la conceptualización de los modelos a los que dan pie. Finalmente, se 
realizará una pincelada de otros modelos de carrera para dar cuenta de las constantes que presentan los desarrollos profesionales de la mujer, independientemente del ámbito en el que se enmarquen.

Una primera tipología remite al esquema clásico de desarrollo profesional masculino, que sitúa su epicentro en el deseo de satisfacer las expectativas profesionales de asumir nuevos retos y/o de ocupar cargos de dirección o cargos de responsabilidad. La literatura señala que este tipo de trayectorias se concretan con transiciones de tipo lineal en una misma organización (Huberman, Thompson y Weiland, 2000), o interorganizacionales, saltando de una organización a otra (Latack, 1984), en función de que permitan satisfacer las expectativas profesionales de ocupar cargos de dirección. En su mayoría, los nuevos retos u oportunidades son brindadas por los mentores; estas figuras suelen ser hombres que las ayudan en su promoción laboral y a quienes ellas atribuyen buena parte de su éxito profesional (Kaufmann, 2008). En términos de las transiciones que configuran este modelo, se habla de transiciones a categorías u organizaciones profesionales más ajustadas a los intereses actuales de la persona (Greenhaus y Callanan, 2006) y, en términos de modelo de carrera, se conceptualiza como cazar oportunidades (Selva, 2012).

Una segunda tipología remite a las trayectorias que no han podido desarrollarse o, en caso de haberse desarrollado, han sido para desestabilizarse. Según Selva (2013), este tipo de trayectorias y su paralización se explican, entre otras causas, por los cambios experimentados en la organización. Estos cambios pueden ir desde una fusión a una re-estructuración, pasando por un cambio en la línea de mando de la organización. En consonancia con otras aportaciones (Greenhaus y Callanan, 2006; Freixas, 2004), esta agrupación o modelo de desarrollo se caracteriza por un tipo de transiciones a ninguna parte enmarcadas en un entorno hostil, y se caracteriza por la soledad y la falta de apoyo institucional. Esta agrupación se asemeja a las transiciones que Huberman et al. (2000) tildan como sin salida y se conceptualiza, en términos de modelo de carrera, como callejón sin salida (Selva, 2012).

Una tercera tipología sitúa su epicentro en la comodidad profesional. Este modelo se ajusta a mujeres que encuentran su principal satisfacción y realización fuera del ámbito profesional, considerando el trabajo sólo como un medio de vida. Si, como dice De la Garza (2000), el trabajo tiene un substrato objetivo más primario, como la alimentación, y otro subjetivo más personal que implica la satisfacción de necesidades sociales, las mujeres bajo este modelo buscarían satis- 
facer sus necesidades objetivas, encontrando la realización de las subjetivas en su espacio personal. Este modelo deja caducos algunos estereotipos socialmente enraizados que presentan a la mujer, como al hombre, con el anhelo o deseo natural por ocupar posiciones de dirección (Selva y cols., 2012). En relación a otras clasificaciones, este modelo se correspondería con la regresión de la que hablan Huberman et al. (2000), entendida no como la pérdida de desarrollo profesional, sino como la ganancia en la vida personal. En términos de modelo de carrera, se conceptualiza como Mi vida está en otro lugar (Selva y cols., 2012).

La cuarta y última tipología que configura un modelo remite a las trayectorias que se desarrollan en una misma organización y en las que las mujeres sienten la necesidad de devolver la confianza que sus empresas, previamente, han depositado en ellas. Es por ello que su desarrollo profesional suele acompañarse de una actitud de espera constante respecto a la aparición de nuevas oportunidades a las que puedan ser invitadas. Bajo este supuesto, las mujeres experimentarían transiciones dependiendo del entorno que las rodea (la organización), del apoyo que la misma empresa les ofrece como muestra de agradecimiento por su compromiso organizacional (Blau, 1985) y por los valores de entrega, honestidad y fidelidad que ellas han demostrado previamente (Greenhaus y Callanan, 2006; Freixas, 2004). Esta agrupación se asemeja a las transiciones de estabilización frecuentemente primeriza (Super, 1963; 1976), y se correspondería con el modelo Todo por mi organización (Selva, 2012).

A pesar de encontrar clasificaciones y agrupaciones distintas de la carrera profesional de la mujer, vemos que existen puntos de convergencia entre ellas -su esencia- que permiten subsumirlas en cuatro grandes modelos. Más allá del ámbito profesional, hay otros modelos de carrera que explican la trayectoria femenina, por ejemplo, en el ámbito deportivo. Este es el caso de los modelos desarrollados por Pallarés, Azócar, Torregrosa, Selva y Ramis (2011). Estos autores nos presentan tres tipos de trayectorias femeninas: la lineal, que se corresponde con la dedicación exclusiva al deporte; la convergente, en la que el deporte es prioritario pero se compatibiliza con una formación alternativa; y la paralela, en la que el deporte y la formación superior reciben la misma priorización.

Es interesante destacar este modelo deportivo porque su clasificación, a pesar de las evidentes diferencias que presenta con el modelo profesional, hace patente una constante en la trayectoria femenina: el 
escaso o nulo tiempo que destinan las mujeres a cultivar otras actividades fuera de su espacio personal o profesional (léase también deportivo), como, por ejemplo, aprender inglés o tocar la guitarra. Estas actividades pueden ser clave para la promoción profesional y la atención a las redes organizacionales (Davidson y Cooper, 1992), y pueden explicar ciertas limitaciones experimentadas.

Volviendo al ámbito profesional, a modo y semejanza al desarrollo propuesto por Super (1963; 1976), Kaufmann (2008) propone dos agrupaciones de desarrollo profesional de la mujer que permiten atender a las limitaciones experimentadas (según su autora, estas limitaciones se presentan inalterables en los diferentes momentos profesionales). Bajo este supuesto, encontramos la primera agrupación, compuesta por mujeres con trayectorias largas y que han llegado a posiciones de dirección; y la segunda, compuesta por mujeres con trayectorias incipientes o recién ingresadas al mundo laboral.

Las primeras, en su desarrollo profesional, han tenido que batallar con estereotipos de género asociados a altas posiciones, compatibilizar los espacios sin renunciar al personal -en pro de ser madres y simultáneamente desarrollar una carrera profesional plena-, y aluden como principal limitación a la gestión de sus anhelos de cambio. Es decir, los cambios que pueden producirse respecto a sus propias voluntades o anhelos -por ejemplo, en relación a querer tener hijos- a los que el entorno, fuertemente condicionado por una cultura organizacional predominantemente masculina, responde de una forma marcadamente hostil.

Por el contrario, las segundas ven su vida profesional y familiar como una proposición dicotómica de sí o no. Esta dicotomía les hace renunciar, a menudo, a la maternidad, aparcando este deseo en pro de realizar una carrera profesional exitosa. Las mujeres que se encuentran en estos momentos de trayectoria tienen una necesidad constante de aprobación, a pesar de ser altamente competitivas, y acusan como principal limitación la falta de modelos femeninos.

\section{CONSIDERACIONES FINALES}

Las agrupaciones o modelos de carrera citados muestran como constante la limitación manifiesta que la mujer mantiene respecto a la conciliación de los diferentes espacios, sin olvidar la relativa a la cultura 
y socialización organizativa, ampliamente citadas en los referentes literarios (Chao, O'Leary-Kelly, Wolf, Klein y Gardner, 1994; Wajcman, 1998). Ambas limitaciones no son más que las dos caras de una misma situación. A modo de ejemplo, en la segunda agrupación de trayectoria de Kaufmann (2008), comprendida por mujeres con trayectorias incipientes o recién ingresadas en el mundo laboral, la principal limitación que se acusa es la falta de referentes femeninos. Si bien es cierto que hay modelos, como es el caso de sus madres, éstos están caducos y ya no son válidos para los momentos actuales. Esta falta de referentes femeninos en el mundo laboral nos lleva a pensar en la necesidad de construir una identidad de trabajo ligada al género femenino o, lo que es lo mismo, fomentar una contra-cultura femenina independiente de la masculina, actual e imperante en el mundo laboral (Beriain, 2000). Esta contracultura ayudaría a dinamitar viejas creencias acerca del trabajador ideal: entendido como aquél que trabaja a tiempo completo, sin tiempo para la maternidad y la crianza de los hijos (Williams, 2000), que siguen llevándonos, sistemáticamente, a perpetuar la reproducción homosocial en las estructuras organizativas e institucionales.

Sean modelos, clasificaciones o agrupaciones de trayectoria (Huberman et al., 2000; Kaufmann, 2008; Selva, 2018; Super, 1963; 1976), los diferentes autores y sus estudios demuestran que: a) no hay trayectorias idénticas, pero sí semejanzas en las transiciones experimentadas, los entornos que las rodean y los apoyos institucionales recibidos; y b) las trayectorias y transiciones profesionales siempre van acompañadas de ciertas dosis reflexivas por parte de las protagonistas -acerca de su historia personal y profesional- (Schlossberg, Waters y Goodman, 1995). Estas semejanzas, como ha podido verse, pueden recogerse en cuatro grandes modelos de carrera que comparten características comunes, o esencias, y dan respuesta a la realidad del desarrollo laboral de la mujer hacia cargos de dirección.

Si el objetivo de este artículo era conocer los modelos, trayectorias o mapas de ruta que permiten a la mujer llegar a los cargos de dirección, los resultados esbozados: a) rompen con la creencia de que la mujer desea llegar a ocupar posiciones de dirección como si de un anhelo innato se tratase; y b) dan luz a los diferentes caminos que la llevan a estas posiciones, desbancando caducas creencias de modelos únicos escalares. Los estudios, desde diferentes enfoques y ámbitos disciplinares, demuestran que son varias las rutas trazadas hacia cargos de dirección, en las que son características ciertas secuencias de desa- 
rrollo profesional (Freixas, 2004; Super 1962) y desde las que se comparten ciertos elementos nucleares, como los factores contextuales, experienciales o reflexivos.

Los resultados de este artículo sugieren que es necesario estudiar desde más ópticas, aproximaciones y variabilidad de casos los modelos de carrera. Esto permitirá detectar y dar fiabilidad a los trayectos observados, legitimar limitaciones recurrentes y facilidades encontradas, así como mostrar una fotografía nítida del fenómeno que permita esbozar líneas de intervención conexas a la realidad y en pro de un futuro más igualitario en las bases y cúspides organizativas a través de una de sus vías de acción: la adecuada gestión del talento y de la diversidad.

\section{REFERENCIAS}

Agut, S. y Hernández, M. (2007). Factores que dificultan el acceso de las mujeres a puestos de responsabilidad: una revisión teórica. Apuntes de Psicología, 25, 201-214. Disponible en: http://copao.cop. es/files/contenidos/VOL25_2_7.pdf.

Baltres-Löhr, C. (2006). Instrument for changing gender inequalities in scientific careers. Women in Scientific Careers Unleasing the Potential. OCDE, 167-178.

Barberá, E., Ramos, A. y Sarrió, M. (2000). Mujeres directivas ante el tercer milenio: el proyecto NOWDI XXI. Papeles del Psicólogo, 75, 4652. Disponible en: http://www.redalyc.org/html/778/77807508/.

Baron, R. A. y Byrne, D. (2005). Prejuicio: causas, efectos y formas de contrarrestarlo. En R. A. Baron y D. Byrnes (Eds.), Psicología social (pp. 215-261). Madrid: Prentice-Hall.

Beriain, J. (2000). El ser oculto de la cultura femenina en la obra de Georg Simmel. Revista Española de Investigaciones Sociológicas, 89, 141182. DOI: $10.2307 / 40184229$.

Bonilla, A. y Martínez, I. (1992). Análisis del currículum oculto de los modelos sexistas. En M. Moreno (Coord.), Del silencio a la palabra. Coeducación y reforma educativa (pp. 60-92). Madrid: Ministerio de Asuntos Sociales, Instituto de la Mujer, Colección Estudios.

Bozeman, B., Dietz, J. S. y Gaughan, M. (2001). Scientific and technical human capital: An alternative model for research-evaluation. International Journal Technology Management, 22(7-8), 716-740. DOI: 10.1504/ijtm.2001.002988. 
Carosio, A. (2010). El trabajo de las mujeres: desigualdad, invisibilidad y explotación. Revista venezolana de estudios de la mujer, 15(35), 7-14. Disponible en: http://www.scielo.org.ve/scielo.php?script=sci_arttextEpid=S1316-37012010000200001.

Cemborain, E. (2007). Techo de cristal y escalera de cristal en las cooperativas de Mondragón. Participación de las mujeres en los órganos de gobierno de las cooperativas. En First International CIRIEC Research Conference on the Social Economy, Victoria, BC, Canadá.

Chao, G. T., O'Leary-Kelly, A. M., Wolf, S., Klein, H. J. \& Gardner, P. D. (1994). Organizational socialization: Its content and consequences. Journal of Applied Psychology, 79(5), 730. DOI: 10.1037//00219010.79 .5 .730 .

Cuadrado, I. y Morales, J. (2007). Algunas claves sobre el techo de cristal en las organizaciones. Revista de Psicología del Trabajo y de las Organizaciones, 23, 183-202. Disponible en: http://www.redalyc.org/ articulo.oa?id=231317597002.

Davidson, M. y Cooper, C. (1992). Shattering the glass ceiling. The Woman Manager. London: Paul Chapman Publishing Ltd.

De la Garza, E. (2000). El papel del concepto de trabajo en la teoría del siglo XX. En De la Garza, E. Tratado latinoamericano de sociología del trabajo. (pp. 15-35). México: Facultad Latinoamericana de Ciencias Sociales.

Dickman, A. e Eagly, A. (2000). Stereotypes as dynamic constructs: Women and men of the past, present, and future. Personality and Social Psychology Bulletin, 26, 1171-1188. DOI: 10.1177/0146167200262001.

Fernandez, R. y Mors, M. (2008). Competing for jobs: Labor queues and gender sorting in the hiring process. Social Science Research, 37, 1061-1080. DOI: 10.1016/j.ssresearch.2007.10.003.

Freixas, M. (2004). La influencia de factores personales, institucionales y contextuales en la trayectoria y el desarrollo docente de los profesores universitarios. Educar, 33, 31-59. Disponible en: http:// educar.uab.cat/article/view/260.

González, A. (2009). La carrera profesional de las investigadoras jóvenes: un camino lleno de posibilidades. Revista iberoamericana de ciencia, tecnología y sociedad, 12(4), 31-54. Disponible en: http://www.scielo.org. ar/scielo.php?script=sci_arttextEpid=S1850-00132009000100003.

Goyette, K. y Mullen, A. (2006). Who Studies the Arts and Sciences? Social Background and the Choice and Consequences of Undergra- 
duate Field of Study. The Journal of Higher Education, 3(77), 497-538. DOI: /10.1080/00221546.2006.11778936.

Greenhaus, J. H. y Callanan, G. A. (2006). Encyclopedia of Career Development. London: Sage Publications.

Grimmer, B. y Röhl, T. (2005). Female students at Universities in BadenWürtteemberg, Rhône-Alpes and Catalonia. A secondary analysis of gender, interest in science and research, and the intention to do a doctorate. Papers, 76, 199-215. DOI: 10.5565/rev/papers/v76n0.1009.

Heilman, M. (2001). Description and prescription: How gender stereotypes prevent women's ascent up the organizational ladder. Journal of Social Issues, 57, 657-674. DOI: 10.1111/0022-4537.00234.

Huberman, M., Thompson, C. L. y Weiland, S. (2000). Perspectivas de la carrera del profesor. En Biddle, B. J.; Good, T. L. y Goodson, I. F., La enseñanza y los profesores I. La profesión de enseñar. Barcelona: Paidós. Instituto Nacional de Estadística (2016). Mujeres graduadas en educación superior. Ministerio de Igualdad. Consultado el 31 de marzo del 2017, en: http://www.ine.es/ss/Satellite?L=es_ESEc=INESeccion_C $\varepsilon \mathrm{cid}=1259925481157 \varepsilon \mathrm{p}=1254735110672 \varepsilon$ pagename=ProductosY Servicios\%2FPYSLayoutEparam3=1259924822888.

Junquera, B. (2004). Factores contextuales, empresariales e intrínsecos a la empresaria y éxito en las empresas propiedad de mujeres: una revisión de la literatura. En Roig, S.; Ribeiro, D.; Torcal V.R.; De la Torre, A. y Cerver, E. (Eds.), El emprendedor innovador y la creación de empresas de I+D+I (pp. 961-985). Universidad de Valencia.

Kaufmann, A. (2008). Women in Management and Life Cycle: Aspects that Limit or Promote Getting to the Top. New York: Palgrave Macmillan.

Latack, J. (1984). Career Transitions within Organizations: An exploratory study of Work, Nonwork and Coping Strategies. Organizational Behavior and Human Performance, 34, 296-322. DOI: 10.1016/00305073(84)90041-2.

Linehan, M. (2000). Senior Female International Managers: Why so few? Ashgate: Aldershot.

López, M. (2007). Factores determinantes en el acceso de la mujer a puestos directivos. Capital Humano, 207, 84-93. Disponible en: https://dialnet.unirioja.es/servlet/articulo?codigo $=2217000$.

López, E. y García-Retamero, R. (2009). Mujeres y liderazgo: ¿discapacitadas para ejercer el liderazgo en el ámbito público? Feminismo/s, 13, 85-104. DOI: 10.14198/fem.2009.13.06. 
Molero, F., Cuadrado, I., García-Ael, C., Recio, P. y Rueda, B. (2009). Mujer y liderazgo en el siglo XXI: Una aproximación psicosocial a los factores que dificultan el acceso de la mujer a los puestos de responsabilidad. Madrid: Instituto de la Mujer.

Moreno, A. (2016). Las mujeres en las organizaciones empresariales: un escenario para el desarrollo de su proyecto profesional y de vida. Tesis Doctoral. Universidad de Sevilla.

Padilla, M. (2001). Barreras y limitaciones en el desarrollo profesional de la mujer. Portularia, 1, 223-232. Disponible en: https://idus.us.es/ xmlui/bitstream/handle/11441/16827/file_1.pdf?sequence $=1$ EisAllowed=y.

Pallarés, S., Azócar, F., Torregrosa, M., Selva, C. y Ramis, Y. (2011). Modelos de trayectoria deportiva en waterpolo y su implicación en la transición hacia una carrera profesional alternativa. Cultura, Ciencia y Deporte, 17(6), 93-103. DOI: 10.12800/ccd.v6i17.36.

Ridgeway, C. (2001). Gender, status, and leadership. Journal of Social Issues, 57, 637-655.

Sarrió, M., Barberá, E., Ramos, A. y Candela, C. (2002). El techo de cristal en la promoción profesional de las mujeres. Revista de Psicología Social, 17, 167-182. DOI: 10.1174/021347402320007582.

Schlossberg, N., Waters, E. y Goodman, J. (1995). Counseling adults in transition: Linking practice with theory. New York: Springer.

Selva, C. (2012). Explicant la propia carrera: una Mirada longitudinal sobre gènere, organitzacions $i$ vida personal en els relats de les trajectòries professionals. Tesis Doctoral. UAB. Disponible en: http://www.tdx.cat/bitstream/ handle/10803/96301/csolde1. pdf?sequence $=1$.

(2013). Models of professional Career of the Managerial Woman. Universitas Psychologica, 12(4), 1237-1254. DOI: 10.11144/javeriana. upsy 12-4. mopc.

(2018). Stories on the path to management. Gender and professional trajectories. Academia Revista Latinoamericana de Psicología, 31(2). En prensa.

Selva, C., Tresserra, O., Pallarès, S. y Sahagún, M. (2012). El río y el sendero como carreras profesionales: contrastando relatos con género. Quaderns de Psicologia, 14(1), 61-70. DOI: 10.5565/rev/qpsicologia. 1102 .

Simó, J. y Casado, V. (2006). Carreras profesionales: ¿algo más que reconocimiento? Atención Primaria, 38(7), 405-408. DOI: 10.1016/ s0212-6567(06)70533-9. 
Simpson, R. (2000). Gender mix and organisational fit: How gender imbalance at different levels of the organisation impacts on women managers. Women in Management Review, 1(15), 5-19. DOI: 10.1108/09649420010310173.

Super, D. (1962). Psicología de la vida profesional. Madrid: Rialp. (1963). Career development: Self-concept theory. New York: College Entrance Examination Board. (1976). Career education and the meanings of work. Washington, D.C.: U.S. Office of Education.

Wajcman, J. (1998). Like a Man: Women and Men in Corporate Management. Cambridge: Polity Press. (2004). Technofeminism. Cambridge:Polity Press. MA.

Wajcman, J. y Martin, B. (2002). Narratives of identity in modern management: the corrosion of gender difference? Sociology, 36(4), 9851002. DOI: $10.1177 / 003803850203600410$.

Williams, J. (2000). Unbending Gender: Why Family and Work Conflict and What to Do About. Oxford: Oxford University Press.

SOBRE LOS AUTORES

Clara Selva Olid (clara.selva@uab.cat) es profesora del Departamento de Psicología Social de la Universidad Autónoma de Barcelona (UAB). Doctora en Psicología Social por la Universidad Autónoma de Barcelona (UAB), sus intereses de investigación se relacionan con temas de trayectoria profesional, género, violencia ocupacional y metodología cualitativa, entre otros (ORCID ID: 0000-0001-7390-9889).

Francisco Javier Ruvalcaba Coyaso (fjruval@correo.uaa.mx) es profesor investigador titular a tiempo completo en la Universidad Autónoma de Aguascalientes y doctor en Psicología por la Universidad de Colima (2008-2011). Forma parte del cuerpo académico "Trabajo y relaciones sociales". Sus líneas de investigación son: identidad profesional, trayectorias laborales y transición escuela-trabajo y modelos matemáticos para la toma de decisiones, que son prioritariamente sus áreas de generación de conocimiento (ORCID ID: 0000-0002-0464-4271). 
